\title{
A Case-Based Tropical Medicine Curriculum for Interdisciplinary Global Health Track Residents
}

\author{
Damon Edgar Webb, Will Northquist and Jenny Baenziger, MD \\ Indiana University School of Medicine and Indiana University Center for Global \\ Health
}

Global health education is essential for equipping physicians to improve population health both at home and abroad. Global health is a multidisciplinary specialty with physicians who come from many backgrounds, making it important to reinforce concepts in its education that might not have been the focus of their previous training. Tropical medicine, and its focus on infectious disease, is one area of global health that many physicians may not have focused on extensively. We developed a curriculum for tropical medicine for residents in the Indiana University Interdepartmental Global Health Track, a 2-3 year co-curricular focus on global health with residents from Family Medicine, Internal Medicine, MedPeds, Pediatrics, OBGYN, EM, and others. Research has shown that casebased learning (CBL) is effective in engaging students and faculty compared to other educational methods. It is believed that utilizing CBL in delivering a tropical medicine curriculum to these residents will develop proficiency in the subject over the 8 sessions developed. Residents will learn about a variety of infectious diseases in each session by working through a case, engaging in critical thinking in small groups, then taking a quiz. Following completion of all sessions, they will be complete a summative test and a survey subjectively assessing the curriculum. We hope this novel curriculum will prove effective in teaching the essentials of tropical medicine to Global Health Track residents and will serve as an example for the development of other tropical medicine curricula. 